\title{
Predictors of 90-Day Restart of Renal Replacement Therapy after Discontinuation of Continuous Renal Replacement Therapy, a Prospective Multicenter Study
}

\author{
Susanne Stads ${ }^{a, b}$ K. Merijn Kant ${ }^{c}$ Margriet F.C. de Jong ${ }^{d}$ Wouter de Ruijter ${ }^{\mathrm{e}}$ \\ Christa M. Cobbaert ${ }^{f}$ Michiel G.H. Betjes ${ }^{g}$ Diederik Gommers ${ }^{a}$ \\ Heleen M. Oudemans-van Straaten ${ }^{\mathrm{h}}$ \\ ${ }^{a}$ Department of Intensive Care, Erasmus Medical Center, Rotterdam, The Netherlands; ${ }^{\text {b }}$ Department of Intensive \\ Care, Ikazia Hospital, Rotterdam, The Netherlands; ' Department of Intensive Care, Amphia Hospital Breda, Breda, \\ The Netherlands; ${ }^{d}$ Department of Nephrology, University Medical Center Groningen, Groningen, The Netherlands; \\ ${ }^{e}$ Department of Intensive Care, Noordwest Ziekenhuisgroep, Alkmaar, The Netherlands; ${ }^{f}$ Department of Clinical \\ Chemistry and Laboratory Medicine, Leiden University Medical Center, Leiden, The Netherlands; ${ }^{9}$ Department of \\ Nephrology, Erasmus Medical Center, Rotterdam, The Netherlands; ${ }^{\text {h }}$ Department of Intensive Care, Amsterdam \\ UMC, Vrije Universiteit Amsterdam, Amsterdam, The Netherlands
}

\section{Keywords}

Acute kidney injury · Discontinuation · Continuous renal replacement therapy · Neutrophil gelatinase-associated lipocalin

\begin{abstract}
Background: Restart of renal replacement therapy (RRT) after initial discontinuation of continuous RRT (CRRT) is frequently needed. The aim of the present study was to evaluate whether renal markers after discontinuation of CRRT can predict restart of RRT within 90 days. Methods: Prospective multicenter observational study in 90 patients, alive, still on the intensive care unit at day 2 after discontinuation of CRRT for expected recovery with urinary neutrophil gelatinase-associated lipocalin (NGAL) available. The endpoint was restart of RRT within 90 days. Baseline and renal characteristics were compared between outcome groups no restart or restart of
\end{abstract}

RRT. Logistic regression and receiver operator characteristic curve analysis were performed to determine the best predictive and discriminative variables. Results: Restart of RRT was needed in 32/90 (36\%) patients. Compared to patients not restarting, patients restarting RRT demonstrated a higher day 2 urinary NGAL, lower day 2 urine output, and higher incremental creatinine ratio (day $2 / 0$ ). In multivariate analysis, only incremental creatinine ratio (day $2 / 0$ ) remained independently associated with restart of RRT (OR $5.28,95 \% \mathrm{Cl}$ $1.45-19.31, p=0.012)$. The area under curve for incremental creatinine ratio to discriminate for restart of RRT was 0.76 (95\% Cl 0.64-0.88). The optimal cutoff was 1.49 (95\% Cl 1.441.62). Conclusion: In this prospective multicenter study, incremental creatinine ratio (day 2/0) was the best predictor for restart of RRT. Patients with an incremental creatinine ratio at day 2 of 1.5 times creatinine at discontinuation are likely to need RRT within 90 days. These patients might benefit from nephrological follow-up.

(C) 2019 The Author(s)

Published by S. Karger AG, Basel

\section{KARGER}

E-Mail karger@karger.com www.karger.com/bpu

\section{(C) 2019 The Author(s)}

This article is licensed under the Creative Commons AttributionNonCommercial-NoDerivatives 4.0 International License (CC BYNC-ND) (http://www.karger.com/Services/OpenAccessLicense) Usage and distribution for commercial purposes as well as any distribution of modified material requires written permission.
Susanne Stads, MD

Department of Intensive Care

Ikazia Hospital Rotterdam, Montessoriweg 1

NL-3083 AN Rotterdam (The Netherlands)

E-Mail s.stads@ikazia.nl 


\section{Introduction}

Acute kidney injury (AKI) is a common complication of critical illness. If renal replacement therapy (RRT) is required, patients have excess mortality even when adjusted for severity of disease [1-4]. Among survivors, renal function might not recover and patients may sooner or later need chronic RRT. Currently, no clinical characteristics or biomarkers are known to predict restart of RRT. Identification of patients at risk and close nephrological follow-up may be important to take preventive measures and restart RRT timely.

Neutrophil gelatinase-associated lipocalin (NGAL) at intensive care unit (ICU) admission has high potential as predictor for AKI [5-11] and need of continuous RRT (CRRT) [9, 12-14]. After renal injury, NGAL is secreted into blood and urine within $2 \mathrm{~h}$ [15], whereas the rise in creatinine takes days. While serum creatinine is a marker of renal function, NGAL reflects renal injury. Evaluation of NGAL at ICU admission as a predictor for dialysis dependency after an episode of AKI has shown disappointing results $[16,17]$. However, different types of NGAL and commercial kits for determination of NGAL are available and therefore results might not be comparable. Only a few studies evaluated NGAL later during ICU admission. In patients after out-of-hospital-cardiac-arrest, NGAL at day 2-3 was better associated with AKI and mortality than NGAL at admission $[5,18]$. In sepsis, NGAL at day 7 was associated with 28-day mortality [12]. However, NGAL after discontinuation of CRRT and its association with restart of RRT was not tested. We previously found that incremental creatinine ratio after discontinuation, but not urinary NGAL was independently associated with short-term successful discontinuation of CRRT (no restart of RRT for 7 days) [19]. However, identification of the patients at risk for long-term restart is also important because these patients need close nephrological follow-up after ICU discharge to take protective measures for the kidney, adjust medications, and delay restart or prepare the patient for chronic dialysis.

The aim of this study was to evaluate whether urinary NGAL, urine output, and incremental creatinine ratio measured after discontinuation of CRRT can predict restart of RRT within 90 days.

\section{Methods}

\section{Study Design}

We performed a prospective multicenter observational study in 4 ICUs in the Netherlands (additional file 1) to evaluate short- term (7-day) [19] and long-term (90-day) predictors of restarting RRT after initial discontinuation for expected renal recovery. $\mathrm{Pa}-$ tients were included from May 2013 until September 2015. The protocol was approved by the medical Ethics Committee of Erasmus Medical Centre and local medical Ethical Committees of participating centers. Written informed consent was obtained from all participants or legal representatives.

\section{Study Endpoint}

The endpoint of study was restart of RRT within 90 days after initial discontinuation of CRRT for AKI.

\section{Patients}

All patients, aged 18 years or older, alive and still on the ICU at day 2 after discontinuation of CRRT for renal reasons with expected renal recovery, excluding patients in whom CRRT was discontinued for logistic reasons, such as a CT scan or operation, patients with known end-stage renal disease (chronic kidney disease [CKD]-5), and those in whom CRRT were discontinued to switch to intermittent hemodialysis, were screened for inclusion. Patients discharged from the ICU before day 2 after discontinuation of CRRT were excluded, because day 2 study variables could not be reliably collected.

\section{Study Protocol and Measurements}

The decision to initiate, discontinue, or restart RRT was made by the attending team of physicians and was not defined by protocol. Reason was that there is no consensus and there are no guidelines for restart of RRT. This decision is generally based on a combination of reasons. CRRT was performed according to the local protocol of the hospital as CVVH or CVVHD, delivered dose was $20-35 \mathrm{~mL} / \mathrm{kg} / \mathrm{h}$. Day 0 was defined as the first 6 a.m. after discontinuation of CRRT. At day 2 after initial discontinuation of CRRT, 3 renal markers were determined: urinary NGAL, urine output and the incremental creatinine ratio between day 2 and 0 (at discontinuation; creatinine day $2 / 0$ ), and the nonrenal sequential organ failure assessment (SOFA) score. The assay for determination of urinary NGAL is specified in additional file 1.

We additionally collected: age, sex, weight, BMI, preadmission estimated glomerular filtration rate (eGFR; calculated with CKDepidemiology collaboration formula [20]), history of CKD and other comorbidities, reason for ICU admission, disease severity scores (Acute Physiology And Chronic Health Evaluation III, Simplified Acute Physiology Score III), cause of AKI, main reason for restart and use of diuretics (day 0-2), radiocontrast agents, or nephrotoxic medication (day $0-7)$. Nephrotoxic medication was scored according to the local pharmacological guide, and a list of this medication is added in additional file 2 .

\section{Statistical Analysis}

Sample size calculation was based on an expected incidence of restart of RRT of $30 \%$ [21]. To evaluate the prediction of the renal markers (urinary NGAL, urine output, and incremental creatinine ratio [day 2/0]), we aimed to include 90 patients using the number of events/10 rule. Patients not restarting RRT were compared to patients restarting temporary or chronic RRT within 90 days. Variables were tested for normal distribution using the Shapiro-Wilk test. Continuous variables are expressed as mean (SD) or median (25th and 75th percentile) and categorical data as number and percentage. Unpaired Student $t$ test, Mann-Whitney U test, or Chisquare test was used, where appropriate. Statistical significance 


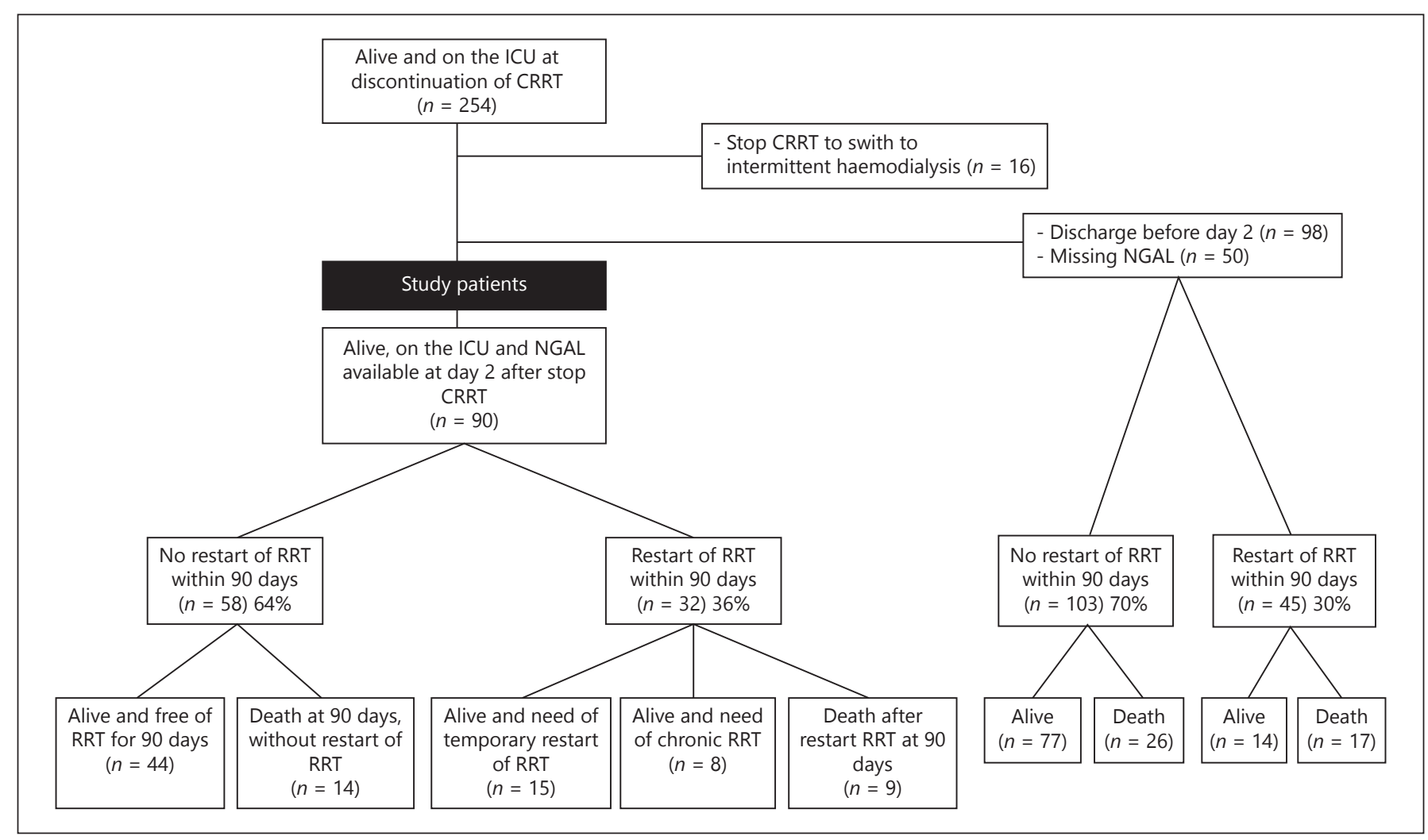

Fig. 1. Flowchart. ICU, intensive care unit; RRT, renal replacement therapy; CRRT, continuous RRT; NGAL, neutrophil gelatinase-associated lipocalin.

was defined as $p<0.05$. To assess the relation between renal markers and other potential predictors of restarting RRT within 90 days, univariate analysis was performed. Subsequently, multivariate analysis was performed, including the 3 above-defined renal markers alone, and after adjustment for preadmission eGFR and nonrenal SOFA score. We also performed a sensitivity analysis including the patients with missing NGAL concentrations and determined the relation between the main reasons for restart and the 3 renal predictors at day 2 . For all analyses, multicollinearity was checked with a maximum variance inflation factor of 10 .

A receiver operator characteristic (ROC) curve was drawn for the best discriminative variable for restart of RRT. The area under the ROC curve (AUROC) was calculated to discriminate for restart of RRT. The Youden index was calculated to determine the optimal cutoff to discriminate for restart of RRT. The 95\% CI for the optimal cutoff was calculated using bootstrapping with 1,000 random samples using the bias corrected and accelerated method.

\section{Results}

Of the 254 patients on the ICU at discontinuation of CRRT, 90 patients were alive, still at the ICU, and had urinary NGAL determined on day 2 after discontinuation of CRRT (Fig. 1). Of these 90 patients, 32 (36\%) patients restarted RRT, while 58 (64\%) did not restart RRT within 90 days after initial discontinuation. Among the 32 patients restarting RRT, 15/32 only temporary needed RRT, whereas $8 / 32$ became dependent of chronic RRT. Nine of these 32 patients died after restarting RRT. About 11/32 patients restarted RRT because of fluid overload, 6/32 restarted RRT because of oliguria, 14/32 restarted RRT because of azotemia or a rise in creatinine, and $1 / 32$ restarted RRT because of hyperkalemia. Among the 58 patients not restarting RRT 14 patients died within 90 days. The clinical course of the 90 study patients and associated NGAL concentrations are shown in Figure 2. RRT was restarted at a median of 4 (3-10) days, range $2-50$. In $11 / 32$ patients, RRT was restarted $>7$ days after discontinuation of CRRT, and in 6/32 patients, RRT was restarted after ICU discharge (range 1-41 days after ICU discharge). The cumulative number of patients restarting RRT after initial discontinuation of CRRT is shown in Figure 3. In the majority of patients, RRT was restarted within 18 days. Among the 148 excluded patients (because of discharge from the ICU before day 2 after dis- 
Fig. 2. Clinical course of the study patients and associated NGAL concentrations. NGAL, neutrophil gelatinase-associated lipocalin, NGAL results in median (25th75th percentile). NGAL is expressed in $\mathrm{ng} /$ $\mathrm{mL}$. ICU, intensive care unit; RRT, renal replacement therapy; NGAL, neutrophil gelatinase-associated lipocalin.

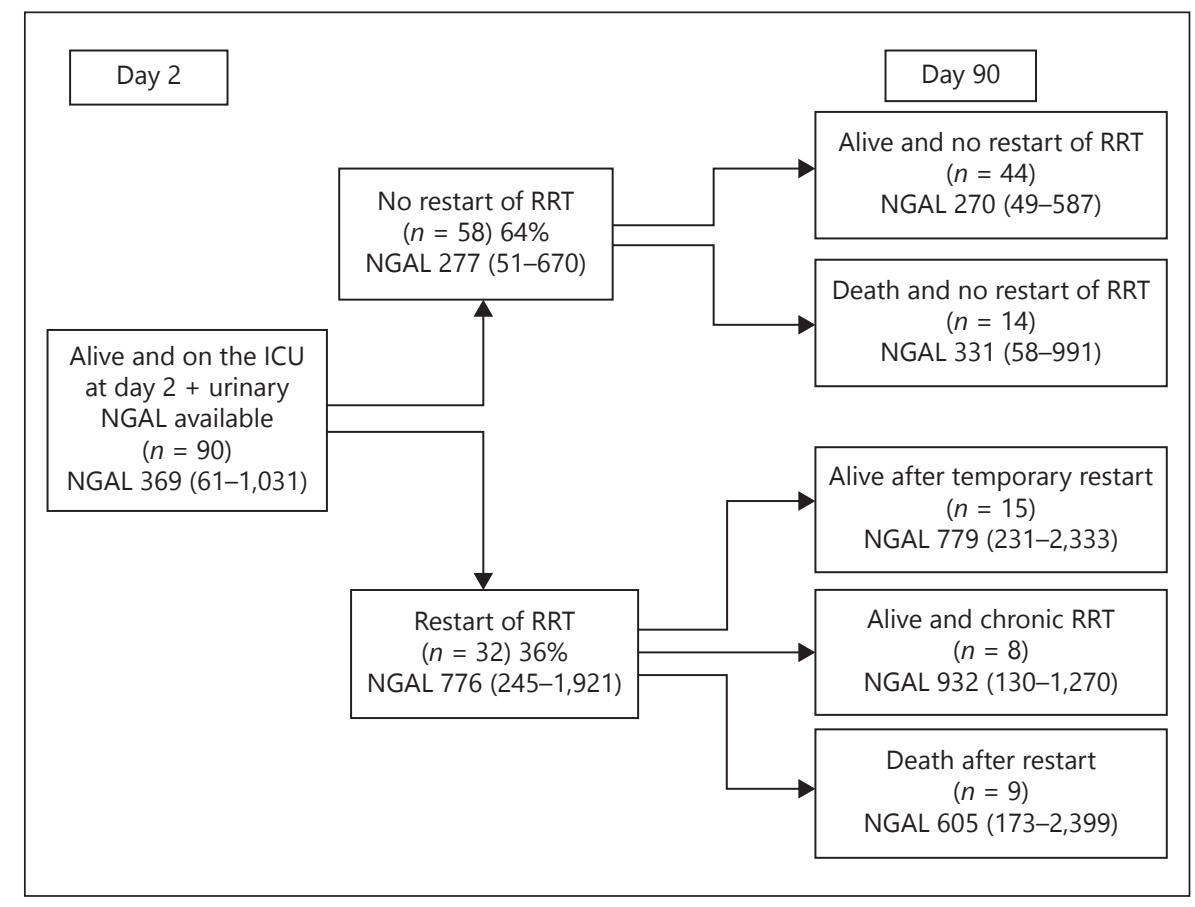

continuation of CRRT [ $n=98$ ] or because of missing NGAL $[n=50]), 45$ (30\%) restarted RRT within 90 days.

To assess characteristics of patients restarting RRT, this group was compared to patients not restarting RRT within 90 days after initial discontinuation of CRRT. Characteristics of these groups are presented in Table 1 and were not different.

Potential Predictors of Restarting RRT within 90 Days after Initial Discontinuation of CRRT

Compared to patients not restarting RRT, patients restarting RRT had a higher day 2 urinary NGAL 776 (245$1,921)$ vs. $277(51-670) \mathrm{ng} / \mathrm{mL}, p=0.005$, a lower day 2 urine output $1.783(1.263)$ vs. 2.503 (1.231) L, $p=0.015$ and higher incremental creatinine ratio (day 2/0) 1.64 $(1.52-1.75)$ vs. $1.26(1.01-1.46), p<0.001$. Day 2 nonrenal SOFA score and preadmission eGFR were not significantly different between groups (Table 2).

\section{Regression Analysis of Potential Predictors of \\ Restarting RRT}

In univariate regression analysis, we found a significant association between restarting RRT within 90 days and lower day 2 urine output (OR 0.60, 95\% CI 0.39-0.92, $p=0.020$ ), higher incremental creatinine ratio (day $2 / 0$; OR 5.15, 95\% CI 1.42-18.69, $p=0.013$ ), and a trend for higher day 2 urinary NGAL (OR 1.00, 95\% CI 1.00-1.00, $p=0.053$; Table 3 ).
In multivariate regression analysis, only incremental creatinine ratio (day 2/0) remained significantly associated with restarting RRT within 90 days (OR 5.28, 95\% CI 1.45-19.31, $p=0.012$; Table 4a). We performed a sensitivity analysis also including the patients with missing urinary NGAL. Among the 140 patients in this group, 52 patients (37\%) restarted RRT, 88 patients $(63 \%)$ did not restart RRT. Also in this analysis only incremental creatinine ratio (day $2 / 0$ ) remained significantly associated with restarting RRT within 90 days (OR 5.45, 95\% CI 1.87-15.88, $p=0.002$; Table $4 \mathrm{~b})$.

Furthermore, we analyzed the association between day 2 predictors urine output, urinary NGAL, and creatinine ratio (day $2 / 0$ ) stratified per reason for restart of RRT (fluid overload, oliguria, azotemia, or creatinine rise and hyperkalemia). We only found an independent association between restart because of oliguria and day 2 urine output (OR 0.040, 95\% CI 0.002-0.678, $p=0.026$ ).

\section{Discrimination of Incremental Creatinine Ratio (Day} 2/0) for Restart of RRT

The AUROC curve for incremental creatinine ratio (day 2/0) to discriminate for restart of RRT within 90 days was 0.76 (95\% CI 0.64-0.88; Fig. 4). The optimal cutoff of the incremental creatinine ratio at day 2 to predict restart of RRT was 1.49 (95\% CI 1.44-1.62), sensitivity 0.79 , and specificity 0.79 . 
Fig. 3. Cumulative number of patients restarting RRT. CRRT, continuous renal replacement therapy.

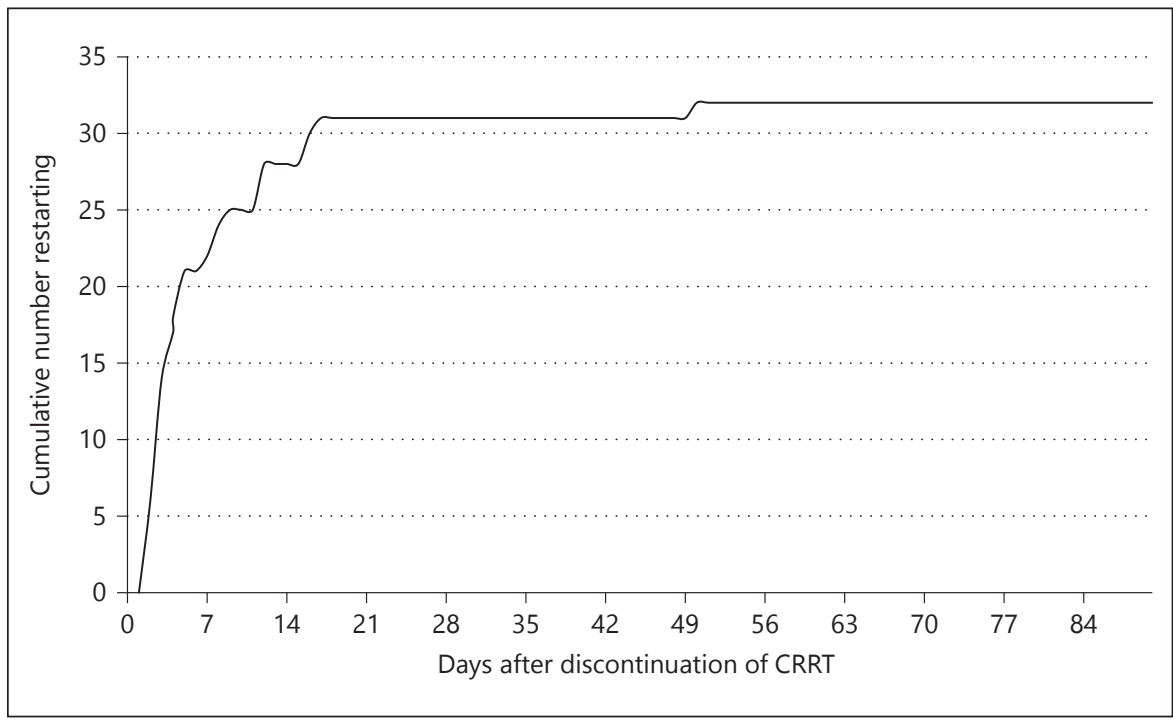

Table 1. Patient and disease characteristics of the patients according to restart of RRT within 90 days or not

\begin{tabular}{|c|c|c|c|}
\hline & $\begin{array}{l}\text { No restart of } \\
\text { RRT }(n=58)\end{array}$ & $\begin{array}{l}\text { Restart of } \\
\text { RRT }(n=32)\end{array}$ & $p$ value \\
\hline Age, years & $61(51-70)$ & $60(15)$ & 0.610 \\
\hline Gender, male, $n(\%)$ & $39(67)$ & $21(66)$ & 0.876 \\
\hline Weight, kg & $81(14)$ & $83(19)$ & 0.646 \\
\hline $\mathrm{BMI}, \mathrm{kg} / \mathrm{m}^{2}$ & $25.4(23.1-29.8)$ & $26.6(5.7)$ & 0.830 \\
\hline History kidney disease, $n(\%)$ & $11(19)$ & $11(34)$ & 0.103 \\
\hline Hypertension, $n(\%)$ & $19(33)$ & $9(28)$ & 0.649 \\
\hline Diabetes mellitus, $n(\%)$ & $8(14)$ & $6(19)$ & 0.535 \\
\hline History of malignancy, $n(\%)$ & $16(28)$ & $8(25)$ & 0.722 \\
\hline History of cardiovascular disease, $n(\%)$ & $21(36)$ & $15(47)$ & 0.323 \\
\hline History of pulmonary disease, $n(\%)$ & $13(22)$ & $11(34)$ & 0.219 \\
\hline \multicolumn{4}{|l|}{ Reason ICU admission, $n(\%)$} \\
\hline Postoperative & $18(31)$ & $12(37)$ & 0.684 \\
\hline Respiratory failure & $8(14)$ & $4(13)$ & \\
\hline Sepsis & $8(14)$ & $7(21)$ & \\
\hline Post cardiac arrest & $8(14)$ & $4(13)$ & \\
\hline Cardiac failure & $9(15)$ & $4(13)$ & \\
\hline Other & $7(12)$ & $1(3)$ & \\
\hline \multicolumn{4}{|l|}{ Cause of AKI, $n(\%)$} \\
\hline Sepsis & $16(27)$ & $13(41)$ & 0.103 \\
\hline Intrinsic & $0(0)$ & $2(6)$ & \\
\hline Toxic & $3(6)$ & $2(6)$ & \\
\hline Ischemic/other & $39(67)$ & $15(47)$ & \\
\hline SAPS III at ICU admission & $52(16)$ & $56(17)$ & 0.313 \\
\hline APACHE III at ICU admission & $87(32)$ & $88(36)$ & 0.901 \\
\hline Diuretic use day $0-2$ & $44(76)$ & $25(81)$ & 0.607 \\
\hline Contrast or nephrotoxic medication day $0-7$ & $54(93)$ & $28(88)$ & 0.359 \\
\hline
\end{tabular}

Mean (SD) for normally distributed variables, median (25th-75th percentile) for non-normally distributed variables.

BMI, body mass index; ICU, intensive care unit; SAPS III, simplified acute physiology score; APACHE III, acute physiology and chronic health evaluation score. 
Table 2. Comparison of potential predictors according to restart within 90 days or not

\begin{tabular}{|c|c|c|c|}
\hline & No restart of RRT $(n=58)$ & Restart of RRT $(n=32)$ & $p$ value \\
\hline \multicolumn{4}{|l|}{ Study variables } \\
\hline Day 2 urinary NGAL $(n=90), \mathrm{ng} / \mathrm{mL}$ & $277(51-670)$ & $776(245-1,921)$ & 0.005 \\
\hline Day $2 / 0$ incremental creatinine ratio $(n=81)$ & $1.26(1.01-1.46)$ & $1.64(1.52-1.75)$ & $<0.001$ \\
\hline \multicolumn{4}{|l|}{ Confounders } \\
\hline Day 2 nonrenal SOFA $(n=82)$ & $4(2-6)$ & $5(3)$ & 0.883 \\
\hline \multicolumn{4}{|c|}{$\begin{array}{l}\text { Median (25th-75th percentile) for continuous variables, number (percentage) when appropriate. } \\
\text { NGAL is expressed in ng/mL. } \\
\text { RRT, renal replacement therapy; NGAL, neutrophil gelatinase-associated lipocalin; SOFA, sequential organ failure assessment; } \\
\text { GFR, estimated glomerular filtration rate; CKD-EPI, chronic kidney disease-epidemiology collaboration. }\end{array}$} \\
\hline
\end{tabular}

Table 3. Univariate analysis of potential predictors of restart of RRT within 90 days

\begin{tabular}{llll}
\hline & OR & $95 \%$ CI & $p$ value \\
\hline Urinary NGAL (day 2), ng/mL & 1.00 & $1.00-1.00$ & 0.053 \\
Urine output (day 2), L & 0.60 & $0.39-0.92$ & 0.020 \\
Creatinine ratio (day 2/0) & 5.15 & $1.42-18.69$ & 0.013 \\
Non-renal SOFA score (day 2) & 0.98 & $0.84-1.15$ & 0.828 \\
Preadmission eGFR & 1.00 & $0.98-1.02$ & 0.883 \\
\hline
\end{tabular}

The ORs are per unit increase.

NGAL is expressed in $\mathrm{ng} / \mathrm{mL}$.

RRT, renal replacement therapy; NGAL, neutrophil gelatinaseassociated lipocalin; SOFA, sequential organ failure assessment; eGFR, estimated glomerular filtration rate.

\section{Discussion}

In this prospective multicenter observational study, we found that after initial discontinuation of CRRT for expected renal recovery, the incremental creatinine ratio at day 2 after discontinuation was the only renal marker that independently predicted restart of RRT within 90 days. Urine output and urinary NGAL on day 2 were no independent predictors. This association remained after adjustment for severity of other organ failure and preadmission renal function, which were not related to restart of RRT within 90 days. With an AUROC of 0.76 , the discrimination of the incremental creatinine ratio (day $2 / 0$ ) was good. A rise of creatinine by one and a half at day 2 after discontinuation appeared as the optimal cutoff to predict restart of RRT within 90 days.
The main reasons for restart were fluid overload, azotemia, or rise in creatinine and oliguria. When analyzing whether the reason for restart was associated with one of the described predictors, we found that only restart because of oliguria was associated with urine output at day 2 , while none of the other reasons were associated with the day 2 renal predictors. However, the robustness of this post hoc analysis can be questioned because subgroups were small. Furthermore, we could only analyze the main reason for restart, while restart of RRT is generally determined by a combination of factors. Big data analysis would be needed to analyze the different combinations of reasons to restart.

The present study shows that two-thirds of the patients restarted RRT within the first week after discontinuation for expected renal recovery and the majority restarted in the first 3 weeks. This finding is in line with previous studies [22, 23]. Interestingly, in our short-term restart study [19], the incremental creatinine ratio also appeared as best predictor for restart within the first week. In that study, we found that the incremental creatinine ratio was as good as creatinine clearance, of which the measurement is more complex.

Creatinine ratio is presently used to classify the severity of AKI according to the kidney disease: improving global outcomes guidelines during its development [24]. In these guidelines, AKI stage 1 is defined as a creatinine ratio from 1.5 to 1.9 times baseline. We found that 2 days after discontinuation, a creatinine of 1.5 times creatinine at discontinuation was the optimal cutoff to predict restart of RRT, which is similar to the creatinine ratio used to define AKI stage 1. Creatinine ratio after discontinuation of CRRT might therefore be a new and simple tool to 
Table 4.

a Multivariate analysis of variables associated with restart of RRT within 90 days $(n=90)$

\begin{tabular}{llll}
\hline & OR & $95 \%$ CI & $p$ value \\
\hline Step 1 & & & \\
$\quad$ Urinary NGAL (day 2) & 1.00 & $1.00-1.00$ & 0.724 \\
$\quad$ Urine output (day 2) & 0.77 & $0.47-1.25$ & 0.286 \\
$\quad$ Creatinine ratio (day 2/0) & 3.94 & $1.03-15.00$ & 0.045 \\
Step 2 & 0.75 & $0.47-1.19$ & 0.221 \\
$\quad$ Urine output (day 2) & 4.05 & $1.06-15.41$ & 0.040 \\
$\quad$ Creatinine ratio (day 2/0) & & & 0.012 \\
Step 3 & 5.28 & $1.45-19.31$ &
\end{tabular}

The ORs are per unit increase. Variables included: primary analysis: urinary NGAL (day 2), urine output (day 2 ), creatinine ratio (day 2/0) as confounders: preadmission eGFR, nonrenal SOFA score (day 2).

RRT, renal replacement therapy; NGAL, neutrophil gelatinase-associated lipocalin; SOFA, sequential organ failure assessment.

b Multivariate sensitivity analysis of variables associated with restart of RRT within 90 days including the patients with missing NGAL $(n=140)$

\begin{tabular}{lccc}
\hline & OR & $95 \%$ CI & $p$ value \\
\hline Step 1 & & & \\
$\quad$ Urine output (day 2) & 0.79 & $0.53-1.19$ & 0.258 \\
$\quad$ Creatinine ratio (day 2/0) & 4.36 & $1.43-13.33$ & 0.010 \\
Step 2 & 5.45 & $1.87-15.88$ & 0.002 \\
$\quad$ Creatinine ratio (day 2/0) & & & \\
\hline
\end{tabular}

The ORs are per unit increase. Variables included: primary analysis: urine output (day 2), creatinine ratio (day 2/0) as confounders: preadmission eGFR, non-renal SOFA score (day 2).

RRT, renal replacement therapy; NGAL, neutrophil gelatinase-associated lipocalin; eGFR, estimated glomerular filtration rate; SOFA, sequential organ failure assessment.

identify patients at risk for further renal function deterioration and thus for nephrological follow-up.

Unexpectedly, we found no independent association between day 2 urinary NGAL and restart of RRT within 90 days. Although median NGAL concentrations were significantly higher in patients restarting RRT compared to patients not restarting RRT, significance was lost when conventional markers of renal function were included in the analysis. Of interest, we found that neither for shortterm (within 7 days) [19] nor for long-term restart, NGAL was independently predictive of restart. This finding is unexpected and has not been reported before in the literature. As mentioned before, urinary NGAL determined at ICU admission is an early marker of renal injury and associated with development of AKI and need of CRRT [5-15]. Previous studies found a poor association between NGAL on admission and late major adverse kidney

Predictors of Restart of RRT events [17, 25]. However, none of these studies determined NGAL after discontinuation of CRRT. We demonstrated that the renal injury marker urinary NGAL after discontinuation of CRRT was also not independently associated with restart of RRT. In contrast, the incremental creatinine ratio, a simple renal function marker, was the best predictor. Accordingly, the more complex renal function marker kinetic eGFR also was a better predictor of late major adverse kidney events than urinary NGAL [16]. It therefore seems that after discontinuation of CRRT for expected recovery, the remaining renal function is more important to predict whether the kidney will recover without restarting RRT than the renal damage marker NGAL, which better predicts the development of AKI.

Another remarkable result was that preadmission eGFR was not significantly different between patients re- 


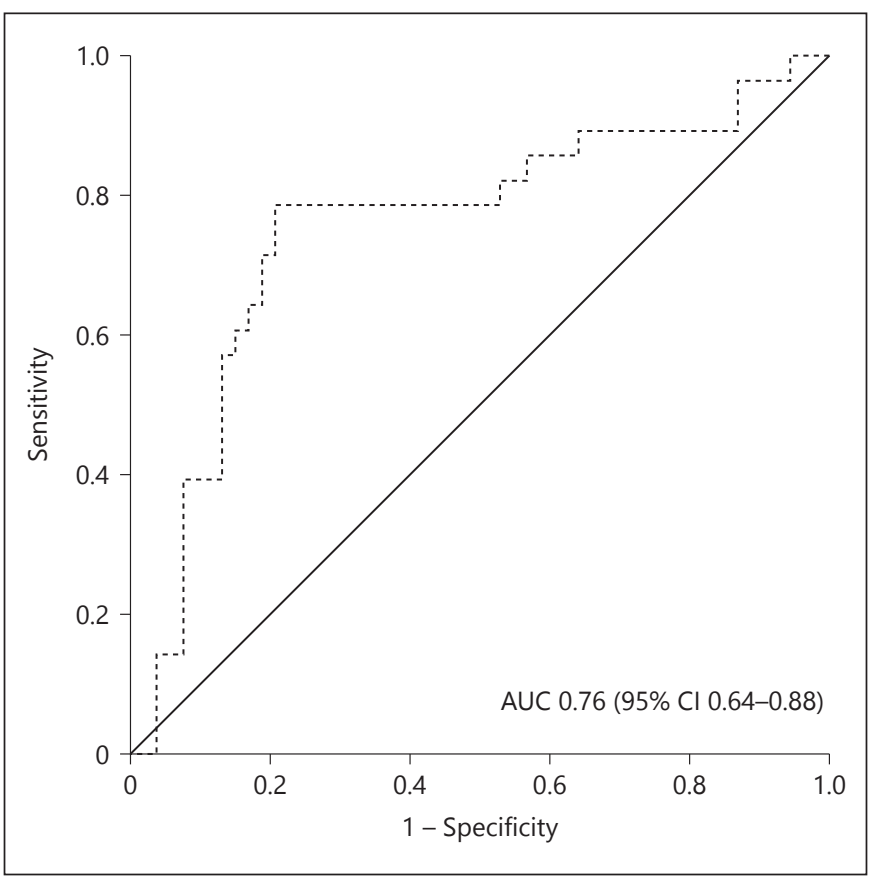

Fig. 4. ROC curve of creatinine ratio (day 2/0) for discrimination of restart of RRT within 90 days. AUC, area under the curve.

starting and patients not restarting RRT. This is in seeming contrast to results found in previous studies in which acute on CKD was a major risk factor for end-stage renal disease $[21,26]$. However, we excluded patients with endstage renal disease (CKD 5) and patients in whom CRRT was discontinued to switch to intermittent hemodialysis. Thus, patients with the worst preadmission renal function were excluded. This may explain why preadmission eGFR did not differ between the need of RRT groups in our cohort. Independent of CKD, severe AKI, that is, AKI requiring RRT is a risk factor for incomplete renal recovery and for the development of subsequent end-stage renal disease and chronic dialysis [27, 28]. However, in our cohort, all included patients had severe AKI because only patients with AKI requiring RRT were included.

Several studies evaluated predictors of restart of RRT or dialysis dependency after AKI. Some studies evaluated risk factors at initiation of CRRT: age, CKD, comorbidities, and severity of disease [21,26,29]. Five studies evaluated renal markers at discontinuation and found urine output, 2-h creatinine clearance, and 24-h urinary creatinine excretion as predictors of successful discontinuation $[22,23,30-32]$. In contrast to the present study, these studies evaluated short-term successful discontinuation (3-15 days). These studies were retrospective in design, included selected populations, such as only surgical pa- tients, and used different modalities of RRT, such as CRRT and intermittent hemodialysis [22, 23, 30-32]. None of these studies evaluated delta creatinine as a predictor. The present prospective study suggests that an incremental creatinine ratio of 1.5 or more 2 days after discontinuation would be a practical trigger to consult the nephrologist for follow-up.

Nowadays, only about $8.5 \%$ of patients at risk for further renal function deterioration after an episode of AKI receive referral to a nephrologist. However, mortality and incidence of end-stage renal disease in this population is high (22\%) [33]. In our cohort, 6/32 patients (19\%) restarted RRT after ICU discharge, especially these patients could benefit from nephrological follow-up, but possibly also those restarting RRT in the ICU. Previous studies found that early nephrological follow-up after an episode of AKI is associated with improved survival [34]. The kidney disease: improving global outcomes guidelines already recommend supportive measures in patients at high risk for AKI or CKD [35]. Therefore, it may be beneficial if intensivists and nephrologists cooperate early after discontinuation of CRRT to determine optimal hemodynamic conditions, dose pharmacotherapy thereby reducing potential nephrotoxic events. Thus, the determination of risk factors for restart of RRT early after discontinuation seems important.

Our study has some limitations. First, a large number of patients were excluded because of discharge before day 2 after discontinuation of CRRT or missing NGAL. This might have caused bias; however, these patients had similar rates of restart, and in the multivariate sensitivity analysis, incremental creatinine ratio also was the only independent predictor. Furthermore, we evaluated the renal markers at day 2 after discontinuation and not at day 1 . The reason was that we aimed to include only patients in whom CRRT was discontinued because of expected recovery of renal function, and we did not want to include patients in whom CRRT was temporarily discontinued because of logistic reasons, such as a CT scan or operation. Furthermore, we evaluated the incremental creatinine ratio day $2 / 0$, because we supposed that the creatinine ratio day $1 / 0$ might not have been discriminative enough. This was later on also found in another study [16]. Second, death might be a competing endpoint. Patients who died within 90 days could have needed RRT if still alive. We therefore presented the NGAL data in all different subgroups. Patients restarting RRT seemed to have higher NGAL concentrations than those who died without receiving RRT. Third, patients who switched directly from CRRT to intermittent hemodialysis were excluded. However, in these patients, RRT 
was not discontinued because of expected recovery, but for change of RRT modality and they therefore did not fulfill the inclusion criteria. Fourth, restart of RRT was not protocolized, and the attending physician decided whether RRT was restarted. Therefore, reasons for restart might be different between centers. However, there is currently no consensus on criteria, and there are no guidelines describing criteria for restart of RRT. Thus, our study describes current practice in 4 Dutch hospitals.

Our study has several strengths. First, we performed a multicenter study, including ICU patients in 4 hospitals, including an academic hospital; hence, our results are highly generalizable. Further, to our knowledge, this is the first prospective study determining predictors for restart of RRT after discontinuation of CRRT, which allows the attending physician to select patients who might especially benefit from nephrological follow-up. Finally, creatinine ratio is a simple marker, which is already used to stage developing AKI and therefore well-known and available in clinical practice.

\section{Conclusions}

In this prospective multicenter study, in patients alive and still on the ICU at day 2 after discontinuation of CRRT, the incremental creatinine ratio at day 2 after discontinuation predicted restart of RRT within 90 days, independent of urinary NGAL, urinary output, preadmission eGFR, and severity of organ failure. The present study suggests that when the rise in creatinine at day 2 after discontinuation of CRRT is 1.5 or more, the patient will likely need restart of RRT within 90 days. We hereby provide a simple and useful tool to select patients who might benefit from nephrological follow-up.

\section{Acknowledgment}

We sincerely regret that Johan Groeneveld who contributed to the concept of this study has died. We miss his sharp and witty research input.

\section{Statement of Ethics}

The protocol was approved by the medical Ethics Committee of the Erasmus Medical Centre and the local medical Ethical Committees of the participating centers. Written informed consent was obtained from all participants or their legal representative.

\section{Disclosure Statement}

The department of intensive care of the Erasmus Medical Centre received an unrestricted research grant from Dirinco. H.O.M.S. is an associate editor of Blood Purification, received speaker's honorary, and participated in advisory meetings from Fresenius, Baxter/Gambro, and Dirinco.

\section{Funding Sources}

The department of intensive care of the Erasmus Medical Centre received an unrestricted research grant from Dirinco.

\section{Author Contributions}

S.S. and M.F.C.J.: contributed to the conception and design of the study. S.S., K.M.K., and W.R.: contributed to the acquisition, analysis and interpretation of data. C.M.C., M.G.H.B., D.G., and H.M.O.S.: contributed to the analysis and interpretation of data. All authors were involved in revision of the manuscript and provided intellectual content of critical importance. H.M.O.S.: supervised the conception and design of the study, analysis and interpretation of data, and supervised drafting of the manuscript. All authors read and gave final approval of the version to be published.

\section{References}

1 Bagshaw SM, Uchino S, Bellomo R, Morimatsu $\mathrm{H}$, Morgera S, Schetz M, et al.; Beginning and Ending Supportive Therapy for the Kidney (BEST Kidney) Investigators. Timing of renal replacement therapy and clinical outcomes in critically ill patients with severe acute kidney injury. J Crit Care. 2009 Mar;24(1):129-40.

2 Bucaloiu ID, Kirchner HL, Norfolk ER, Hartle JE 2nd, Perkins RM. Increased risk of death and de novo chronic kidney disease following reversible acute kidney injury. Kidney Int. 2012 Mar;81(5):477-85.

3 Hoste EA, Bagshaw SM, Bellomo R, Cely CM, Colman R, Cruz DN, et al. Epidemiology of acute kidney injury in critically ill patients: the multinational AKI-EPI study. Intensive Care Med. 2015 Aug;41(8):1411-23.

4 Lin YF, Ko WJ, Chu TS, Chen YS, Wu VC, Chen YM, et al.; NSARF Study Group. The 90-day mortality and the subsequent renal recovery in critically ill surgical patients requiring acute renal replacement therapy. Am J Surg. 2009 Sep;198(3):325-32.

5 Cho YS, Lee BK, Lee DH, Jung YH, Lee SM, Park JS, et al. Association of plasma neutrophil gelatinase-associated lipocalin with acute kidney injury and clinical outcome in cardiac arrest survivors depends on the time of mea- surement. Biomarkers. 2018 Jul;23(5):48794.

6 Dai X, Zeng Z, Fu C, Zhang S, Cai Y, Chen Z. Diagnostic value of neutrophil gelatinase-associated lipocalin, cystatin C, and soluble triggering receptor expressed on myeloid cells-1 in critically ill patients with sepsis-associated acute kidney injury. Crit Care. 2015 May; 19(1):223.

7 de Geus HR, Bakker J, Lesaffre EM, le Noble JL. Neutrophil gelatinase-associated lipocalin at ICU admission predicts for acute kidney injury in adult patients. Am J Respir Crit Care Med. 2011 Apr;183(7):907-14. 
8 Elmedany SM, Naga SS, Elsharkawy R, Mahrous RS, Elnaggar AI. Novel urinary biomarkers and the early detection of acute kidney injury after open cardiac surgeries. J Crit Care. 2017 Aug;40:171-7.

9 Hjortrup PB, Haase N, Wetterslev M, Perner A. Clinical review: predictive value of neutrophil gelatinase-associated lipocalin for acute kidney injury in intensive care patients. Crit Care. 2013 Apr;17(2):211.

10 Mårtensson J, Bell M, Oldner A, Xu S, Venge P, Martling CR. Neutrophil gelatinase-associated lipocalin in adult septic patients with and without acute kidney injury. Intensive Care Med. 2010 Aug;36(8):1333-40.

11 Zhang A, Cai Y, Wang PF, Qu JN, Luo ZC, Chen XD, et al. Diagnosis and prognosis of neutrophil gelatinase-associated lipocalin for acute kidney injury with sepsis: a systematic review and meta-analysis. Crit Care. 2016 Feb;20(1):41.

12 Chang W, Zhu S, Pan C, Xie JF, Liu SQ, Qiu $\mathrm{HB}$, et al. Predictive utilities of neutrophil gelatinase-associated lipocalin (NGAL) in severe sepsis. Clin Chim Acta. 2018 Jun;481: 200-6.

13 Klein SJ, Brandtner AK, Lehner GF, Ulmer H, Bagshaw SM, Wiedermann CJ, et al. Biomarkers for prediction of renal replacement therapy in acute kidney injury: a systematic review and meta-analysis. Intensive Care Med. 2018 Mar;44(3):323-36.

14 Nisula S, Yang R, Kaukonen KM, Vaara ST, Kuitunen A, Tenhunen J, et al.; FINNAKI Study Group. The urine protein NGAL predicts renal replacement therapy, but not acute kidney injury or 90-day mortality in critically ill adult patients. Anesth Analg. 2014 Jul; 119(1):95-102.

15 Bennett M, Dent CL, Ma Q, Dastrala S, Grenier F, Workman R, et al. Urine NGAL predicts severity of acute kidney injury after cardiac surgery: a prospective study. Clin J Am Soc Nephrol. 2008 May;3(3):665-73.

16 Dewitte A, Joannès-Boyau O, Sidobre C, Fleureau C, Bats ML, Derache P, et al. Kinetic eGFR and Novel AKI Biomarkers to Predict Renal Recovery. Clin J Am Soc Nephrol. 2015 Nov;10(11):1900-10.

17 Garcia-Alvarez M, Glassford NJ, Betbese AJ, Ordoñez J, Baños V, Argilaga M, et al. Urinary Neutrophil Gelatinase-Associated Lipocalin as Predictor of Short- or Long-Term Outcomes in Cardiac Surgery Patients. J Cardiothorac Vasc Anesth. 2015 Dec;29(6):1480-8.

18 Beitland S, Waldum-Grevbo BE, Nakstad ER, Berg JP, Trøseid AS, Brusletto BS, et al. Urine biomarkers give early prediction of acute kidney injury and outcome after outof-hospital cardiac arrest. Crit Care. 2016 Oct;20(1):314.

19 Stads SK, Kant KM, de Jong MF, de Ruijter W, Cobbaert CM, Betjes MG, et al. Predictors of short-term successful discontinuation of continuous renal replacement therapy: results from a prospective multicentre study. BMC Nephrol. 2019;20(1):129.

20 Levey AS, Stevens LA, Schmid CH, Zhang YL, Castro AF 3rd, Feldman HI, et al.; CKD-EPI (Chronic Kidney Disease Epidemiology Collaboration). A new equation to estimate glomerular filtration rate. Ann Intern Med. 2009 May;150(9):604-12.

21 Rimes-Stigare C, Frumento P, Bottai M, Mårtensson J, Martling CR, Bell M. Longterm mortality and risk factors for development of end-stage renal disease in critically ill patients with and without chronic kidney disease. Crit Care. 2015 Nov;19(1):383.

22 Fröhlich S, Donnelly A, Solymos O, Conlon $\mathrm{N}$. Use of 2-hour creatinine clearance to guide cessation of continuous renal replacement therapy. J Crit Care. 2012 Dec;27(6): 744.e1-5.

23 Viallet N, Brunot V, Kuster N, Daubin D, Besnard N, Platon L, et al. Daily urinary creatinine predicts the weaning of renal replacement therapy in ICU acute kidney injury patients. Ann Intensive Care. 2016 Dec;6(1):71.

24 Kellum JA, Lameire N; KDIGO AKI Guideline Work Group. Diagnosis, evaluation, and management of acute kidney injury: a KDIGO summary (Part 1). Crit Care. 2013 Feb; 17(1):204.

25 Zeng XF, Li JM, Tan Y, Wang ZF, He Y, Chang J, et al. Performance of urinary NGAL and L-FABP in predicting acute kidney injury and subsequent renal recovery: a cohort study based on major surgeries. Clin Chem Lab Med. 2014 May;52(5):671-8.

26 Forni LG, Darmon M, Ostermann M, Oudemans-van Straaten HM, Pettilä V, Prowle JR, et al. Renal recovery after acute kidney injury. Intensive Care Med. 2017 Jun;43(6):855-66.
27 Rimes-Stigare C, Frumento P, Bottai M, Mårtensson J, Martling CR, Walther SM, et al. Evolution of chronic renal impairment and long-term mortality after de novo acute kidney injury in the critically ill; a Swedish multicentre cohort study. Crit Care. 2015 May; 19(1):221.

28 Stads S, Fortrie G, van Bommel J, Zietse R, Betjes MG. Impaired kidney function at hospital discharge and long-term renal and overall survival in patients who received CRRT. Clin J Am Soc Nephrol. 2013 Aug;8(8):128491.

29 Srisawat N, Murugan R, Lee M, Kong L, Carter M, Angus DC, et al.; Genetic and Inflammatory Markers of Sepsis (GenIMS) Study Investigators. Plasma neutrophil gelatinaseassociated lipocalin predicts recovery from acute kidney injury following community-acquired pneumonia. Kidney Int. 2011 Sep; 80(5):545-52.

$30 \mathrm{Wu}$ VC, Ko WJ, Chang HW, Chen YW, Lin YF, Shiao CC, et al.; National Taiwan University Surgical ICU Acute Renal Failure Study Group (NSARF). Risk factors of early redialysis after weaning from postoperative acute renal replacement therapy. Intensive Care Med. 2008 Jan;34(1):101-8.

31 Jeon J, Kim DH, Baeg SI, Lee EJ, Chung CR, Jeon $\mathrm{K}$, et al. Association between diuretics and successful discontinuation of continuous renal replacement therapy in critically ill patients with acute kidney injury. Crit Care. 2018 Oct;22(1):255.

32 Uchino S, Bellomo R, Morimatsu H, Morgera S, Schetz M, Tan I, et al. Discontinuation of continuous renal replacement therapy: a post hoc analysis of a prospective multicenter observational study. Crit Care Med. 2009 Sep; 37(9):2576-82.

33 Siew ED, Peterson JF, Eden SK, Hung AM, Speroff T, Ikizler TA, et al. Outpatient nephrology referral rates after acute kidney injury. J Am Soc Nephrol. 2012 Feb;23(2):30512.

34 Harel Z, Wald R, Bargman JM, Mamdani M, Etchells E, Garg AX, et al. Nephrologist follow-up improves all-cause mortality of severe acute kidney injury survivors. Kidney Int. 2013 May;83(5):901-8.

35 KDIGO Board Members. Kidney Int Suppl (2011). 2012;2(1):3. 